\title{
SIMULATION OF PEDESTRIAN DETECTION IN URBAN ENVIRONMENT
}

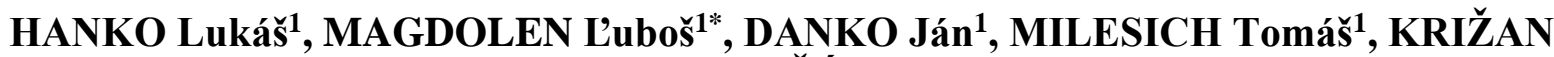 \\ Peter $^{1}$, SKYRČÁK Robert ${ }^{1}$ \\ ${ }^{1}$ Slovak University of Technology in Bratislava, Faculty of Mechanical Engineering, \\ Nám. Slobody 17,812 31 Bratislava, Slovakia, e -mail: lubos.magdolen@stuba.sk
}

\begin{abstract}
Autonomous systems have great potential for future transportation. Even though we have third level autonomous vehicles, that are able to move from place A to place B without drivers' intervention in present, we have still a long journey ahead to complete autonomy. In this article we are dealing with analysis of potential of autonomous vehicles in city transportation. According to high density of pedestrians in urban traffic it is very important to note autonomous vehicles safety.
\end{abstract}

KEYWORDS: Autonomous vehicles, safety, ADAS sensors, obstacle detection

\section{Introduction}

Development of autonomous vehicles currently are more and more important not only among automotive experts, but also in the public sector. Research and development of autonomous vehicles is dedicated to many companies such as Tesla, Google, Ford, Volkswagen or Volvo are dedicated their research and development to autonomous vehicles. Thanks to this increased interest, it is possible that there will be fully autonomous vehicles available to the general public in not-so-distant future.

Autonomous vehicles provide several advantages. They reduce the number of accidents, reduce environment pollution, improve mobility and also increase crew comfort. Autonomous vehicles can be used for personal but also public transport in suburban areas. They can also help improve comfort for people with disabilities [1].

Thanks to complex sensor system, such as camera, radar and lidar, the vehicle can track its surrounding environment, capture barriers and adapt its operation to the situation as necessary [2]. Autonomous vehicles can be divided into five levels from level 0 , a vehicle fully controlled by human, to level 5 , when the vehicle is completely independent on the driver and is able to drive itself. However, in commonly available vehicles, due to the financial complexity of equipment, are mostly used the levels 1 and 2. Regarding these levels, we are talking mainly about emergency braking capabilities, blind spot monitoring and lane tracing assist.

The autonomous vehicle navigation includes several main elements. They are perception, location, mapping, route planning, decision making and vehicle control. Perception uses already mentioned sensors. Data from these sensors are processed by location and mapping, allowing the vehicle to detect possible routes. With these data, it is possible to calculate the best possible route with regard to the environmental conditions (weather conditions, traffic signs, road properties, etc.). Finally, based on these data, it is possible to control the vehicle (steering, brake or acceleration pedal, etc.). Of course, when the conditions of the vehicle change, recorded data are also changed and thus the entire cycle of vehicle control returns to perception [3]. 


\section{Radar and Lidar sensors}

Radar, or radio detection and ranging is sensor used in autonomous vehicles for object detection. Radar device can, by using magnetic waves, detect velocity of vehicle and approached object. Velocity measurements provided by radar are more accurate than measurements provided by cameras. In automobile industry radar is used for several applications mainly obstacle detection of distance maintenance between cars.

Radar in autonomous vehicles can be divided in two groups (Fig. 1) - long-range radar (LRR) and short-range radar (SRR)[4].

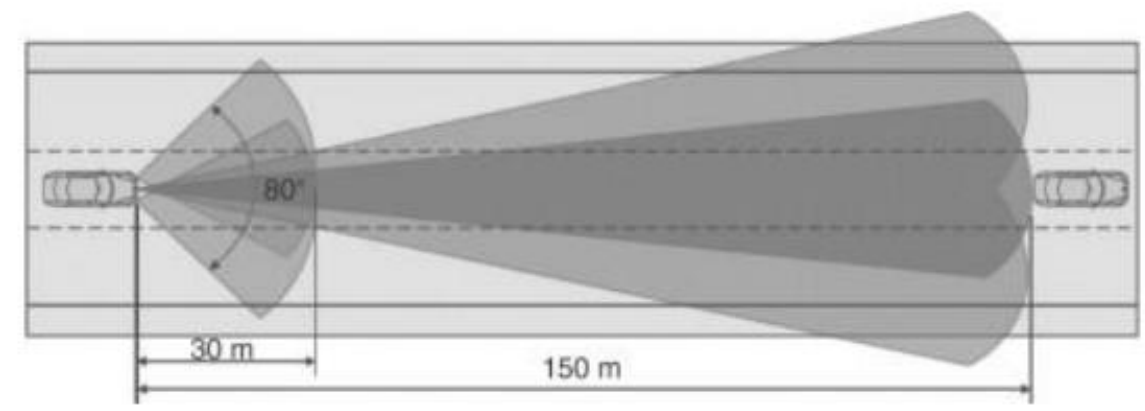

Fig. 1 Long-range radar and Short-range radar

Long-range radar is microwave radar, that measures with frequency from $66 \mathrm{GHz}$ to $77 \mathrm{GHz}$, has low definition, bud it can measure velocity an detect obstacles and cars within $250 \mathrm{~m}$. For example, typical system used in adaptive tempomat has range circa $150 \mathrm{~m}$ and field of sight circa 20 degrees.

Short-range radar is cheaper technology using frequency between $24 \mathrm{GHz}$ and $76 \mathrm{GHz}$. This sensor is used to detect obstacles in short ranges, and is often used as parking assistant. They are preferred because low price and high definition. Because these sensors do not require long range coverage, low frequencies are preferred.

Although radar is more effective than cameras and lidar, in specific situations (for example bad weather) radar has lower beam range and generates less data as lidar. In comparison to cameras and lidar, radar has low definition, especially in vertical direction.

Lidar or light detection and ranging has a wide range of applications for example distance measurement and environmental mapping in autonomous vehicles (Fig. 2).

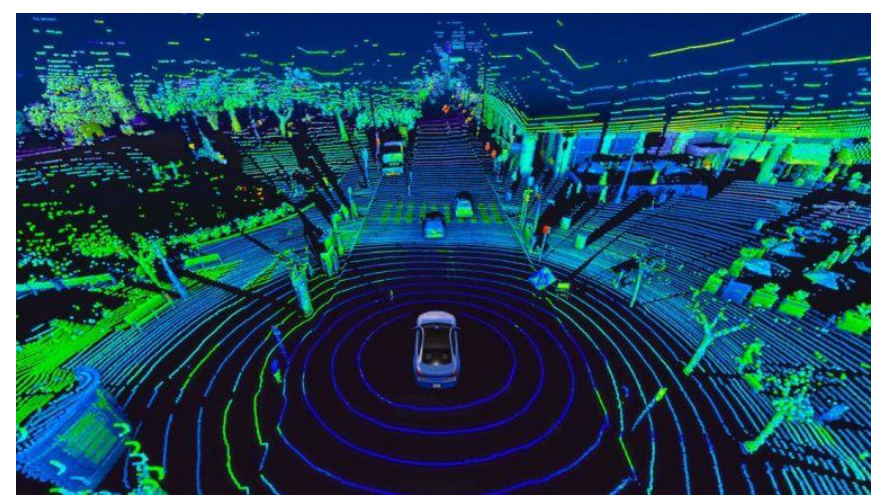

Fig. 2 Lidar mapping

Lidar measures distance to objects in its surrounding. From measured data lidar make a depth map, that is used in the control system of a vehicle. Lidar uses near infra-red light with wave 
length in the range from $800 \mathrm{~nm}$ to $2500 \mathrm{~nm}$ to detect objects in surroundings. Lidar can detect a wide range of targets, including non-metallic objects, rocks, rain, etc. [5].

\section{Obstacle detection}

Safety of autonomous vehicle drive is influenced by many factors. Most common danger is that from surroundings (mostly pedestrians). It would be very dangerous if autonomous system could not stop before pedestrian crossing the road (Fig. 3). Therefore, to prevent these types of accidents, is essential for these systems to detect the obstacle in time. But in real life situations, there are many, hardly predictable, variables that make construction of this type of systems very difficult. Even when systems can detect pedestrian on sidewalk or on crosswalk, it is very difficult to estimate the next steps of pedestrian.

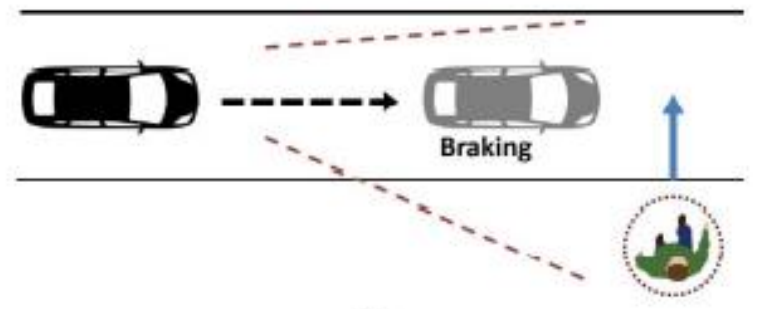

(a)

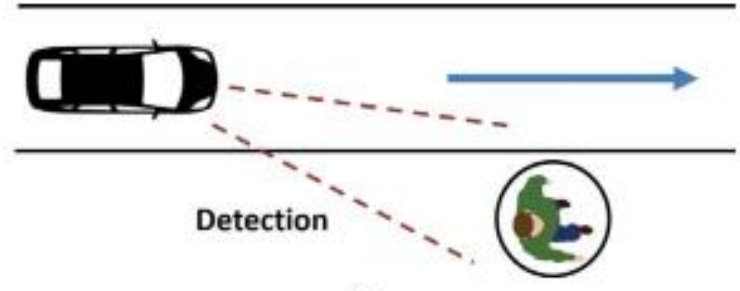

(b)

Fig. 3 Different scenes of pedestrian detection

Figure 3 shows two different scenes of pedestrian detection. In one there is a pedestrian on crosswalk, in the other pedestrian is passing by on sidewalk. In case a) we can see pedestrian stepping on road and the autonomous system detects the obstacle entering its trajectory, so it starts to brake. In case b) the situation is similar, but the pedestrian is walking on the sidewalk and he is not entering the vehicle's trajectory. Autonomous systems detect the pedestrian on the sidewalk and they are ready to activate the breaking maneuver, if the pedestrian enters trajectory of the vehicle [6].

\section{$4 \quad$ Vehicle dynamics}

In order to create a successful simulation in which the autonomous vehicle will detect the obstacle and then start the maneuver, we must create a model of vehicle with its dynamic properties. We have used Simcenter AMESim to create a vehicle dynamic model. AMESim is suitable for modelling physical models. It can offer a perfect simulation platform and flexible solution for mechanisms, hydrokinetics and control systems. The entire modelling and simulation process includes four modes: sketch mode, submodel mode, parameter mode and run mode. The centre of the whole simulation is chassis (Fig. 4). 


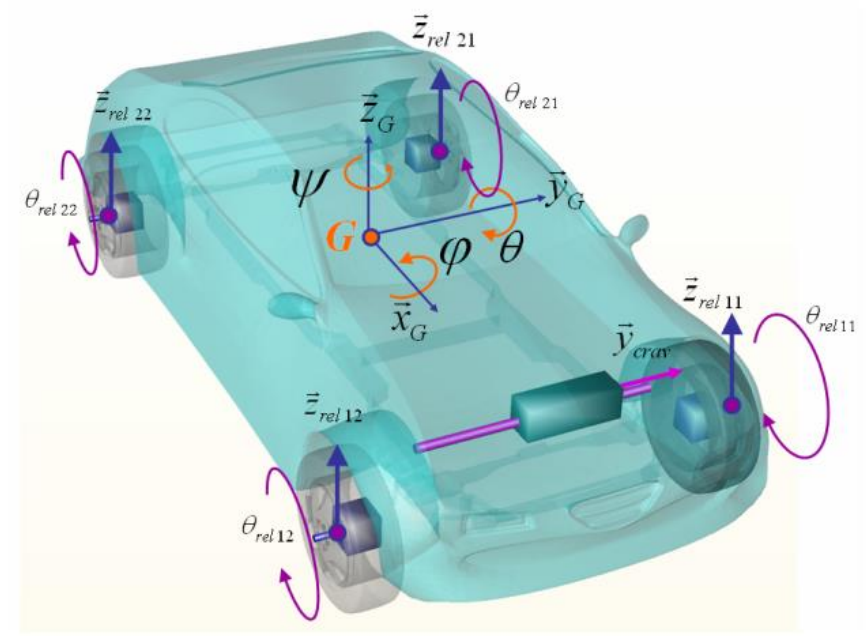

Fig. 4 Vehicle model with 15 DOF

This model is the central module for vehicle dynamics modelling, to which all submodels (damping, silencer, stabilizer, tire, path, sensors, brake, control system, external load, etc.) can be connected. The chassis model, that we used for simulation purposes, is named as VDCAR15DOFKINEXT01. It is designed for longitudinal and lateral dynamics of model. The mechanism described in this model works with 15 degrees of freedom (DOF) and contains 32 ports for subsystem connection. The entire vehicle dynamics scheme (Fig. 5) was created in AMESim in Vehicle dynamics environment, to which interface has been added. Interface can be defined as an interaction site of two systems or parts of those systems. In other words, it is a common boundary of two functional units defined by functional and system properties. Simcenter AMESim - Simulink, and Simulink - Simcenter AMESim interfaces allow to perform simulations by using a combination of models from both software, because two software packages are involved, the interfaces provide two main options: import Simcenter AMESim model to Simulink or import Simulink model to Simcenter AMESim.

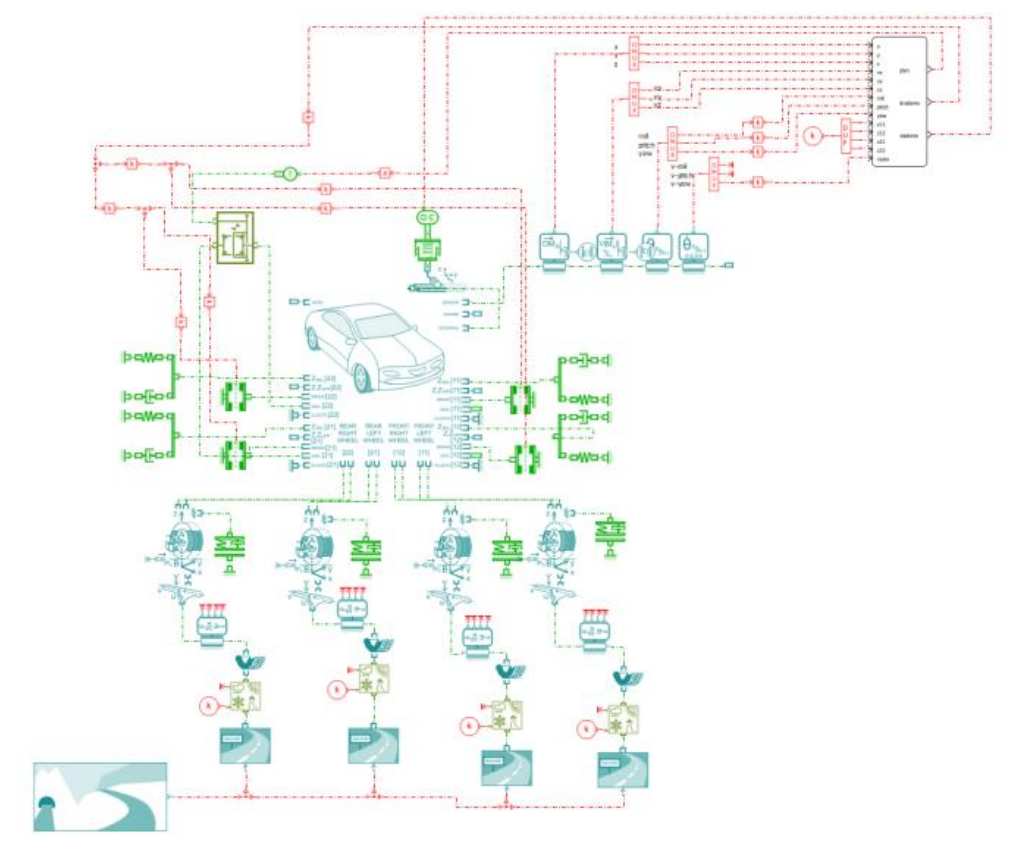

Fig. 5 Vehicle dynamics created in AMESim 


\section{Tire model}

Tires are the most important and the hardest to design components of a car. For vehicle dynamics simulation we used tire model from AMESim library. In this model were pre-entered tire parameters, that were based on mathematical description formulated by Hans Pacejka. This model generates pressing force in dividing line between tire and road. The force is represented by Pacejka formula for combined slip. Tire model (Fig. 6) was imported between tire kinematics model and tire adhesion. Whereas the parameters in this model need to be acquired experimentally, we needed to find the most accurate model in AMESim library. We decided to use tire with 195/50 R15 label, where R15 describes the tire diameter of 15" and the 195 is the width of the tire in millimetres.

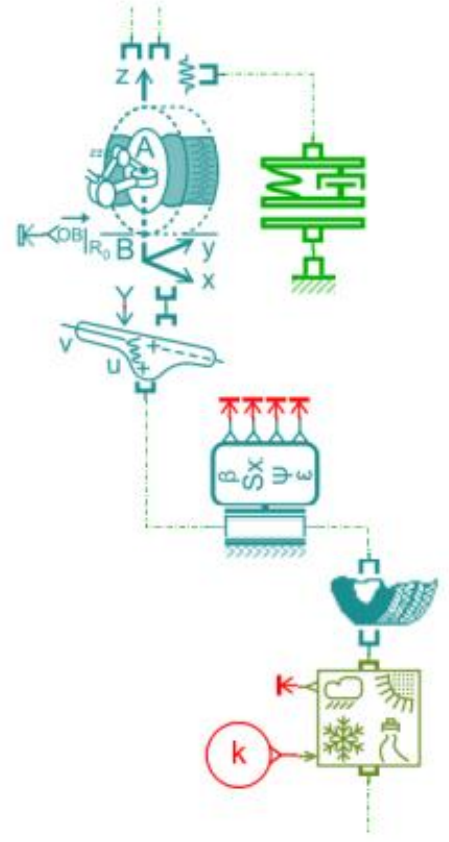

Fig. 6 Tire model

\section{Creating the scenario in Prescan}

For the autonomous vehicle maneuver simulation, scenario needs to be created. This scenario was created in Prescan software. We will create a scenario of emergency braking when detecting an obstacle in the PreScan environment.

This software uses four simple modes (Fig. 7):

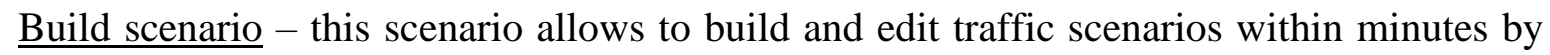
using a road section database, infrastructure components (trees, buildings, traffic signs, etc.), actors (cars, trucks, bikes and pedestrians), weather conditions (rain, snow, fog) and light resources (sun, headlights or lamps). Representation of real roads can be quickly added using Open Street Map, Google Earth, Google 3D Warehouse and/or GPS navigation device.

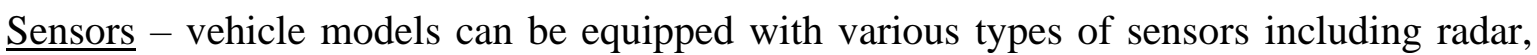
lasers, cameras, ultrasound, GPS, radar or lidar and antennas for communication. Sensor and benchmarking structure are facilitated by simple exchange and modification of sensor and sensor characteristics.

Adding a check system - allows users to design and verify data processing algorithms, collaboration with sensors, decision-making and control, as well as reuse existing Simulink models. 
Starting an experiment - 3D visualization browser allows you to analyse the results of the experiment. It provides multiple aspects, intuitive controls of navigation and image generation and video generation.

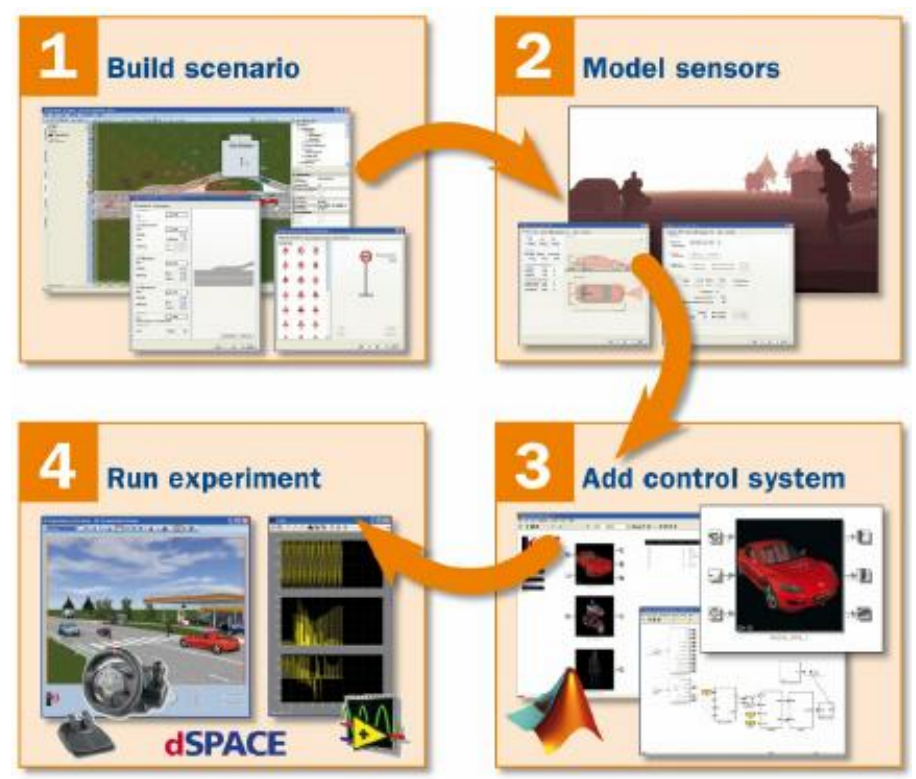

Fig. 7 Prescan application phases

To create a simulation, a few steps were needed. For our experiment a two-lane road was created, and SIMRod vehicle was situated in one lane (Fig. 8), with changing initial velocity. At the same time, velocity of a pedestrian was changing. Simulation was designed so that there was direct contact between vehicle and pedestrian, if there was no radar. The aim of this experiment was to design a system for autonomous vehicle, that can safely stop the vehicle, if there is a pedestrian detected.

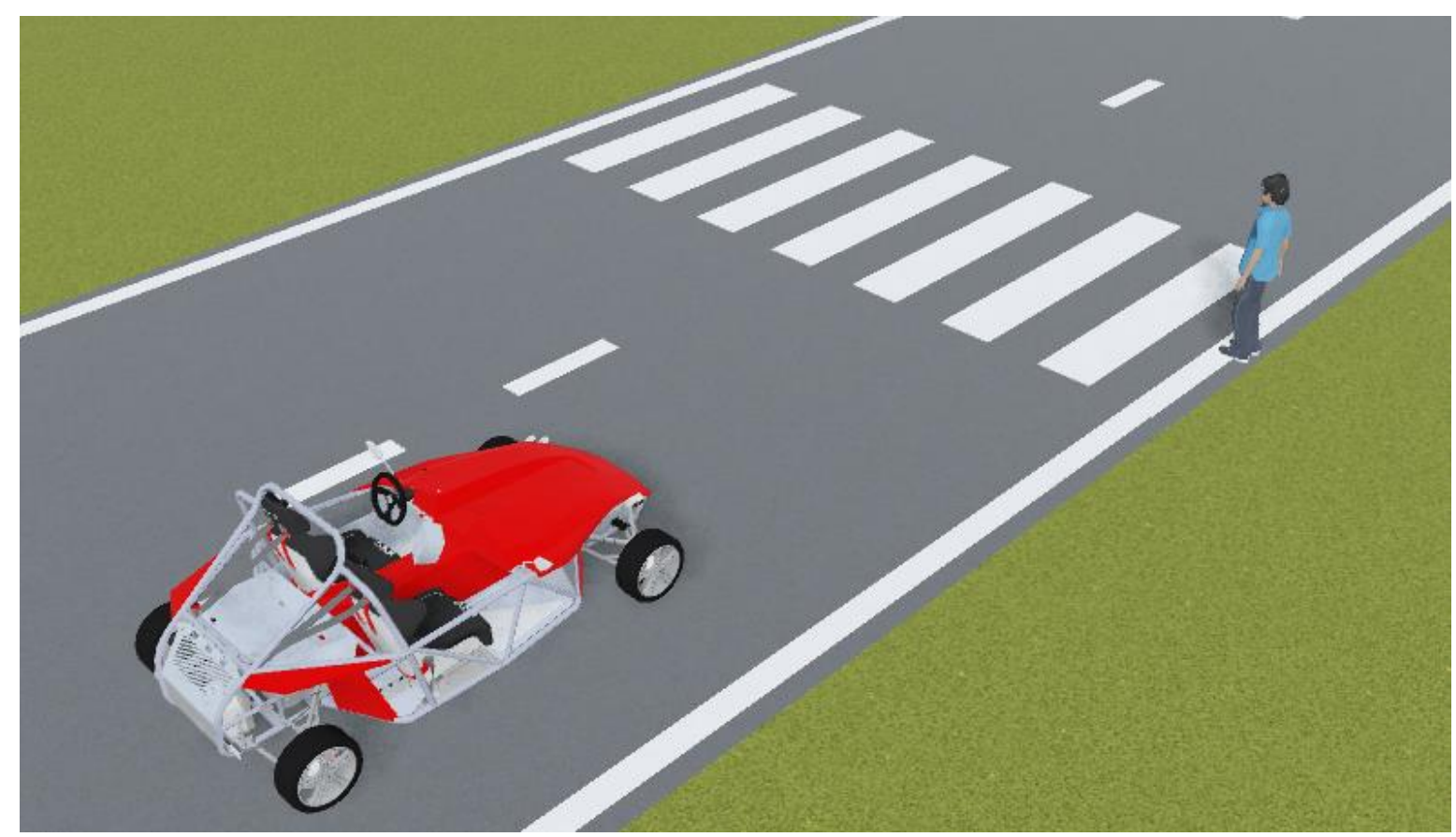

Fig. 8 Scenario created in PreScan 
For the obstacle detection we used two types of radar - long-range radar (LRR) and short-range radar (SRR). Both radars were placed at the front part of the vehicle (Fig. 9) and then, we activated vehicle dynamics, which was replaced in Simulink by vehicle dynamics from AMESim.

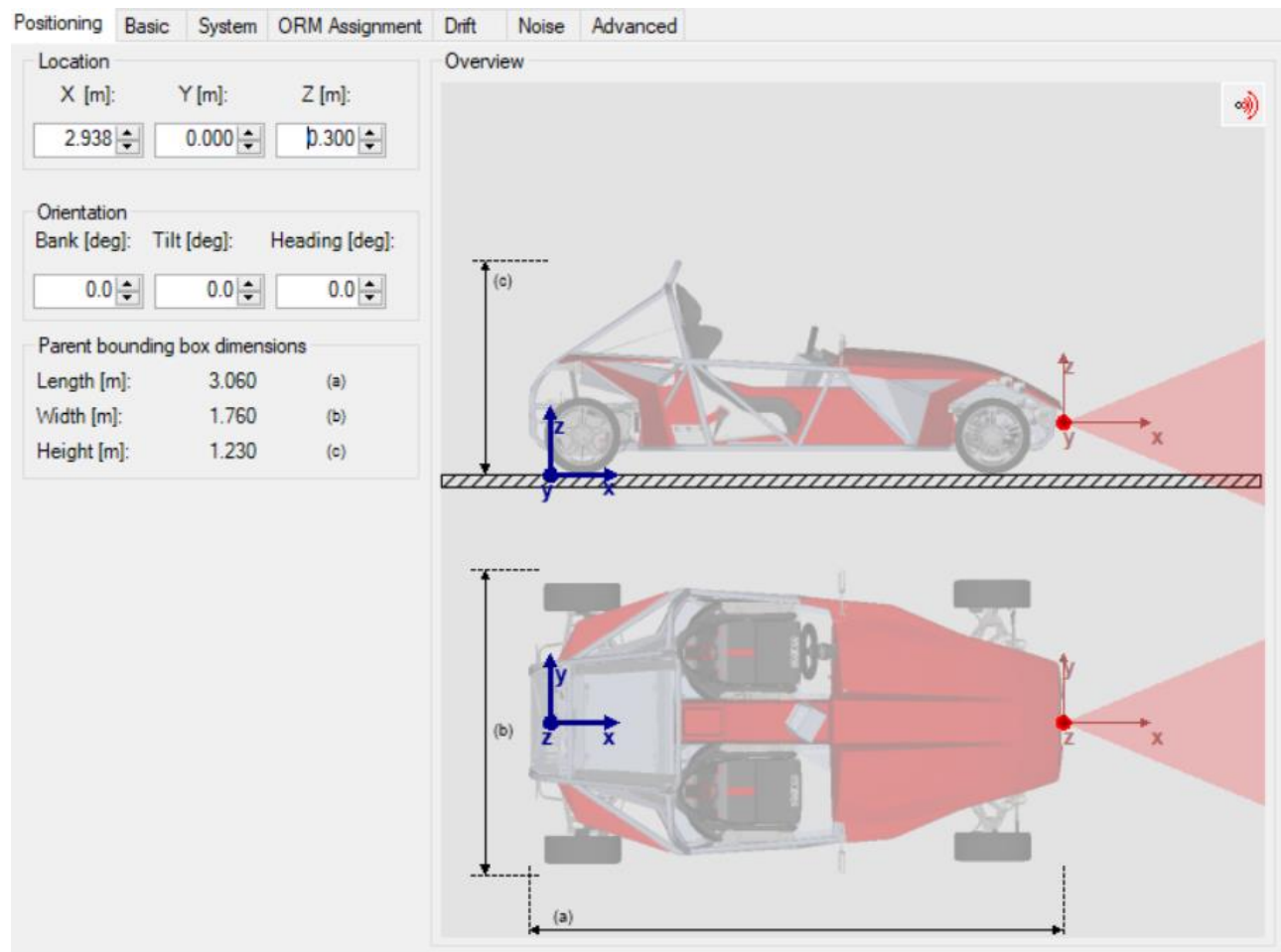

Fig. 9 Radar placement

The aim of this experiment was to design a system, that can provide safe vehicle stopping when obstacle is detected (pedestrian crossing the road), by using the co-simulation of PreScan, AMESim and Matlab/Simulink software. After the scenario was created in PreScan, the whole system was generated in Matlab/Simulink software.

\section{$7 \quad$ Virtual co-simulation of defined scenario}

In this step we created simulation of predefined scenario in PreScan software. In this software we used model of SimRod vehicle and pedestrian model crossing a road. Every actor in this simulation has defined initial velocity, that was changed in specific step. Next, we created sensor models, which was placed on SimRod vehicle. Vehicle dynamics was created in AMESim software and was connected with Matlab/Simulink by interface. After the vehicle dynamics and scenario was created in AMESim or PreScan respectively, we launched individual steps of simulation in Simulink. The simplified scheme of co-simulation can be seen in Figure 10. 


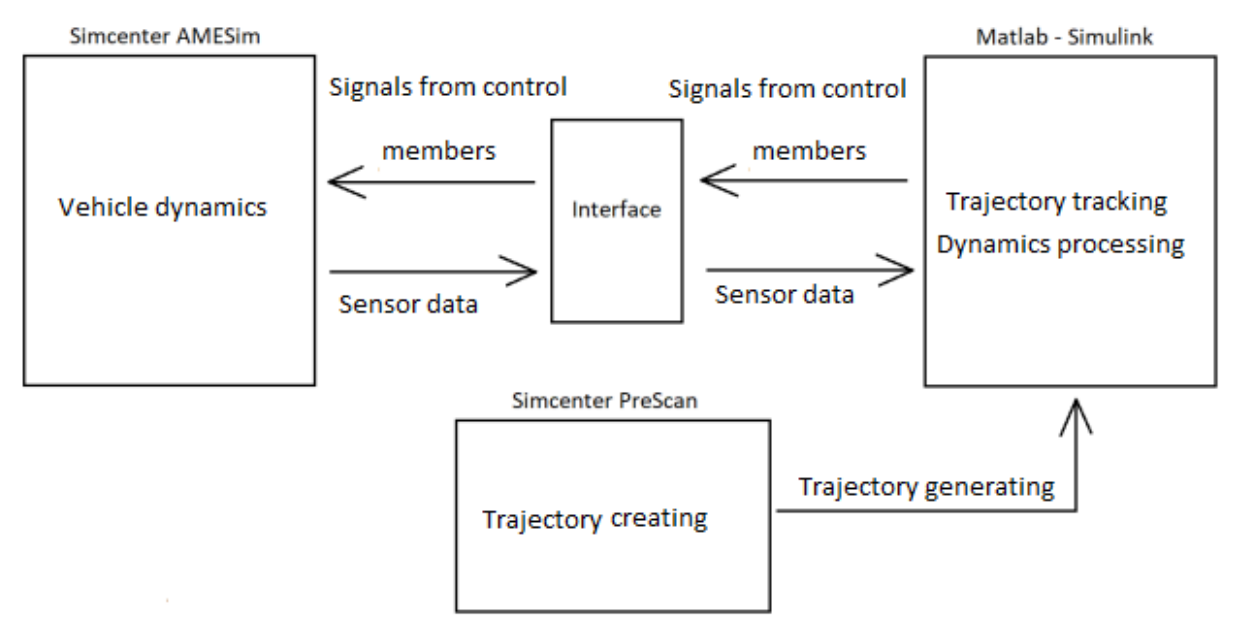

Fig. 10 Co-simulation scheme

\section{RESULTS AND CONCLUSION}

The first scenario, we created was a scenario where autonomous vehicle is, with constant velocity, approaching a pedestrian crossing the road. As we mentioned, we simulated multiple simulations, where we changed initial velocity of the vehicle from $5.5 \mathrm{~m} / \mathrm{s}$ to $16.66 \mathrm{~m} / \mathrm{s}$ with step $2 \mathrm{~m} / \mathrm{s}$, initial velocity of the pedestrian from $0.83 \mathrm{~m} / \mathrm{s}$ to $1.8 \mathrm{~m} / \mathrm{s}$ with step $0.13 \mathrm{~m} / \mathrm{s}$ and the distance between vehicle and crosswalk. The result was limit surface (Fig. 13), that represents border between safe stop and collision. After finding the average pedestrian speed, we can find, with help of this surface, the maximum vehicle velocity for safe stopping. The maximum velocity for autonomous vehicles, according this curve, in highly populated areas is $12.2 \mathrm{~m} / \mathrm{s}$ $(44 \mathrm{~km} / \mathrm{h})$.

We created two scenarios as examples. In the firs scenario initial vehicle velocity is 8.33 $\mathrm{m} / \mathrm{s}$. Pedestrian is detected by set of two radars as an obstacle, and the autonomous vehicle became to brake. Figure 11 shows process of changing velocity and vehicle deceleration. In the time 0.5 seconds we can see the obstacle warning, where systems successfully detected object that appeared in front of the vehicle. Shortly after the obstacle detection the vehicle started to brake. In the next part of the chart, we can see that the vehicle decelerated from $8.33 \mathrm{~m} / \mathrm{s}$ to $1.41 \mathrm{~m} / \mathrm{s}$. Vehicle did not stop entirely because the obstacle moved from radar detection range, and the collision danger was no longer present. After the collision danger was eliminated, vehicle started to increase the velocity. We can see this change at 3.2 seconds. 


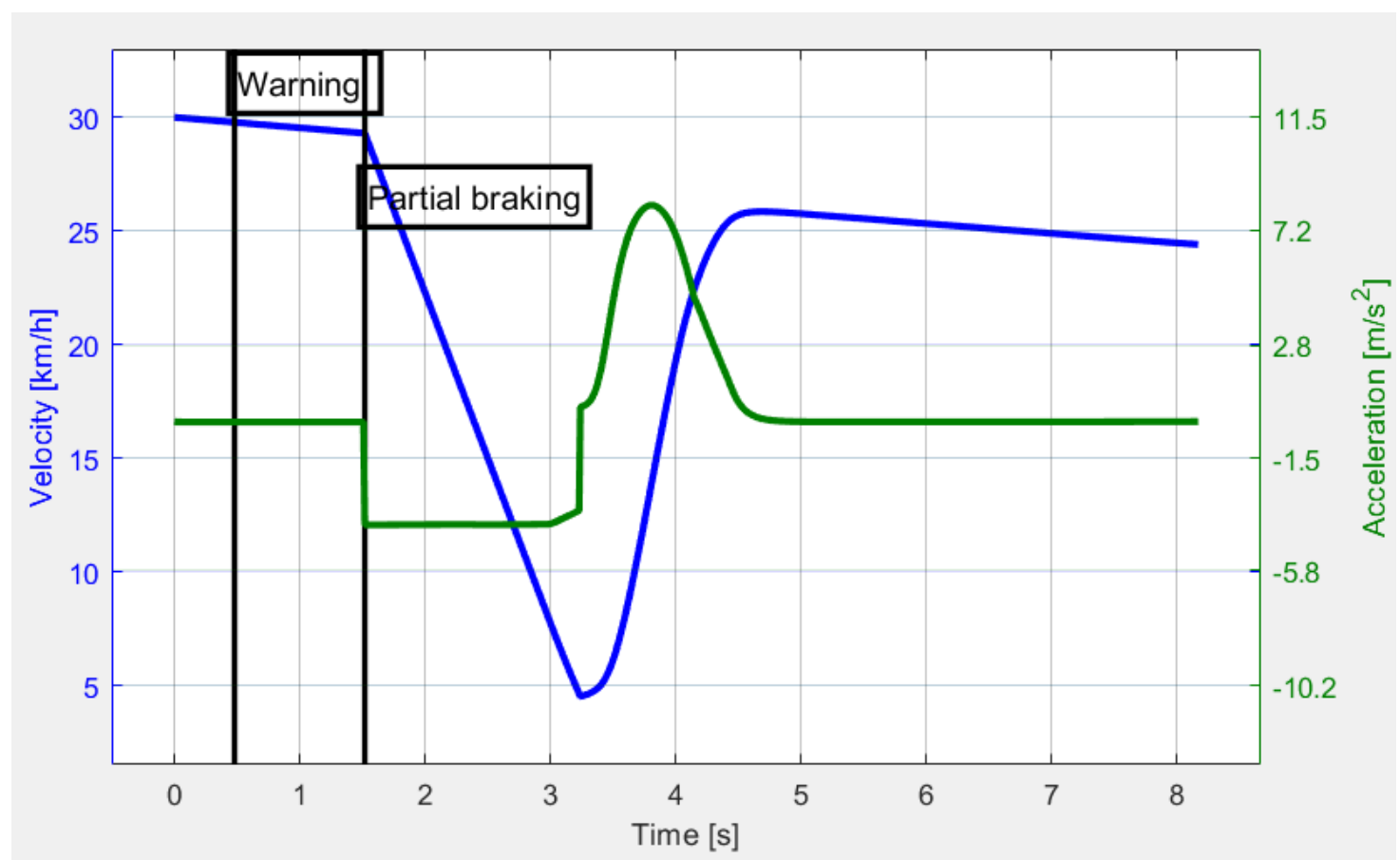

Fig. 11 deceleration at velocity $13.88 \mathrm{~m} / \mathrm{s}$

In the second scenario was similar as the first, but we changed the initial velocity to $19.4 \mathrm{~m} / \mathrm{s}$ $(70 \mathrm{~km} / \mathrm{h})$. Figure 12 illustrates the change of velocity and deceleration of the autonomous vehicle as figure 11, but in this case, we see, that the sensors detected obstacle in almost 8 seconds in simulation. Shortly after obstacle detection the autonomous vehicle started to brake, but in almost 10 second in simulation, we can see the vehicle started to brake more sharply. This is caused by the higher initial velocity and the pedestrian did not have time to pass the road. Systems evaluated the situation as critical and gave the signal to brake sharply to avoid car accident. The vehicle stopped completely before 11 seconds in the simulation. This event is not safe for the pedestrian crossing the road.

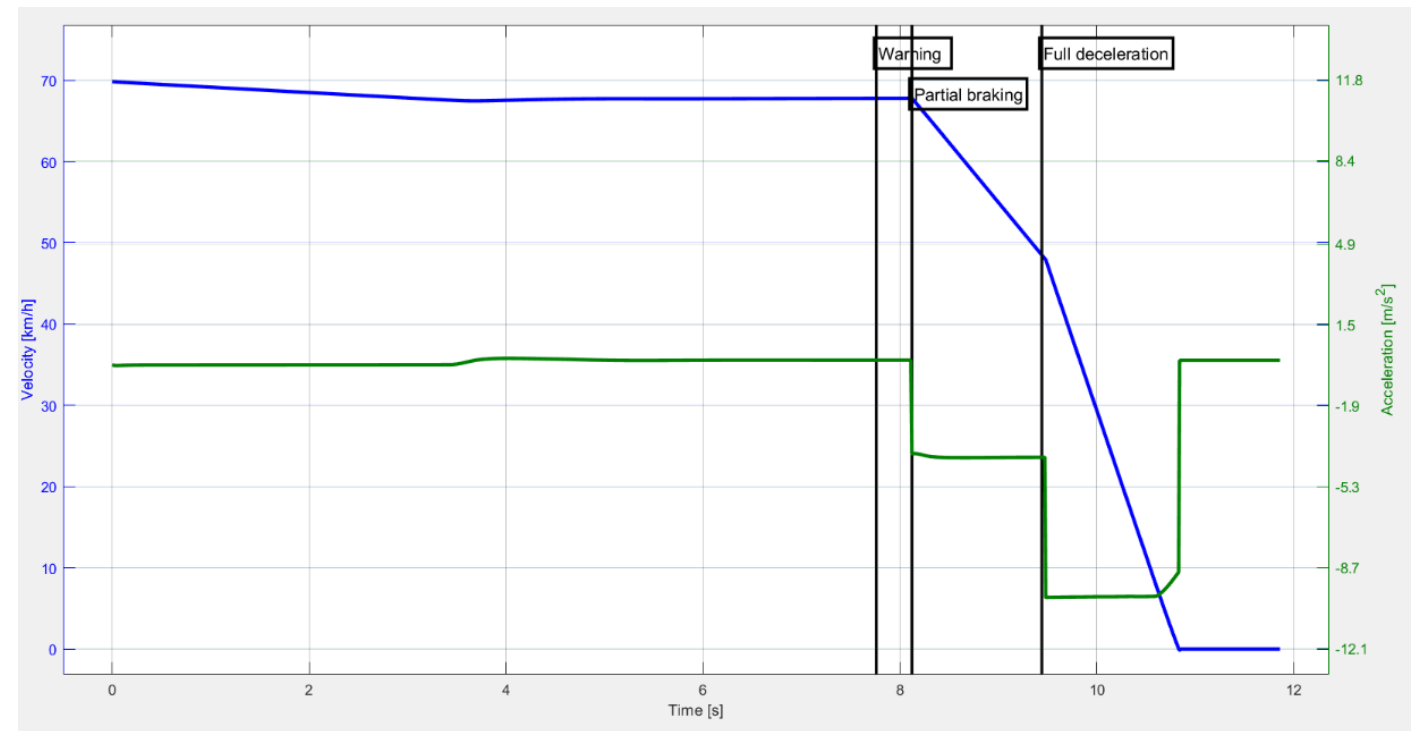

Fig. 12 deceleration at velocity $19.4 \mathrm{~m} / \mathrm{s}$

On Figure 13 we can see so called safe zone, which represents combination of three input factors - vehicle velocity, pedestrian velocity and distance between vehicle and crosswalk, 
that are essential for safe stopping of the vehicle when pedestrian is detected. All values above the limit surface represent collision danger.

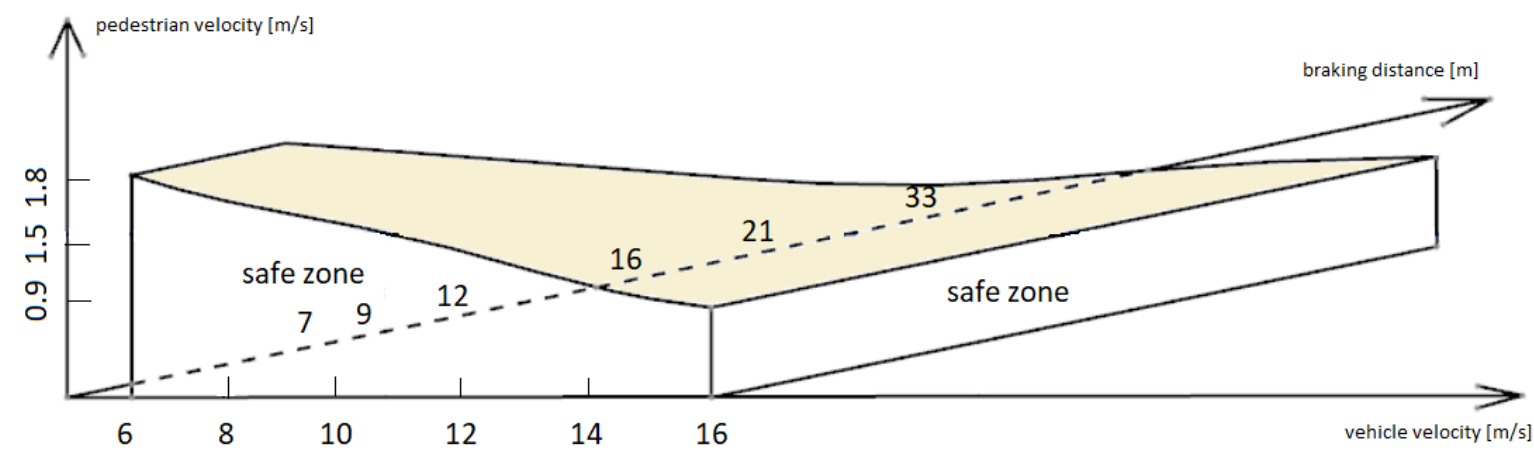

Fig. 13 Limit surface for safe stop and collision

\section{ACKNOWLEDGEMENT}

This research was funded by Slovak Grant Agency APVV-19-0401.

\section{REFERENCES}

[1] Gibson, B. “Analysis of Autonomous Vehicle Policies”, Kentucky Transportation Center, Lexington, Kentucky, 2017. DOI: 10.13023/KTC.RR.2016.29

[2] Liu, J., Sun, Q., Fan, Z., Jia, Y. "TOF Lidar Development in Autonomous Vehicle", In: IEEE $3^{\text {rd }}$ Optoelectronics Global Conference, Shenzhen, China, pp. 185 - 190, 2018. DOI: 10.1109/OGC.2018.8529992

[3] Van Brummelen, J., O'Brien, M., Gruyer, D., Najjaran, H. "Autonomous vehicle perception: The technology of today and tomorrow", Transportation Research Part C: Emerging Technologies, 89 , pp. 384 - 406, 2018. DOI: 10.1016/j.trc.2018.02.012

[4] Meinel, H. H., Bösch, W. "Radar Sensors in Cars", In: Automated Driving, Springer, Basel, Switzerland, pp. 245 - 261, 2017. DOI: 10.1007/978-3-319-31895-0

[5] Bhuiyan, I. K. A. "LiDAR Sensor for Autonomous Vehicle", Technische Universität Chemnitz, Chemnitz, Germany, pp. 5, 2017.

[6] Chae, H., Kang, Ch. M., Kim, B., Kim, J., et al. “Autonomous Braking System via Deep Reinforcement Learning", In: IEEE $20^{\text {th }}$ International Conference on Intelligent Transportation Systems, Yokohama, Japan, pp. $1-6,2017$. DOI: 10.1109/ITSC.2017.8317839 